\title{
Radiological study on mandibular ramus asymmetry in young population
}

\author{
B. Bal, I. Dikbas, O. Malkondu, K. Oral \\ Department of Prosthodontics, Faculty of Dentistry, Yeditepe University, Bagdat, Istanbul, Turkey
}

[Received: 17 November 2017; Accepted: 1 February 2018]

Background: The aim of this study was to estimate the prevalence of ramus asymmetries related to age and gender in a young population and the influence of growth spurt on ramus asymmetry.

Materials and methods: The study consisted of 776 panoramic radiographs of individuals aged 9 to 21 years (335 males and 441 females). Individuals were divided into two groups with respect of linear growth spurt as age 12 in females and age 14 in males. The first group consisted of females aged between 9 and 11 and males between 9 and 13. The second group consisted of females aged between 12 and 21 and males between 14 and 21. Bilateral ramus heights on each radiograph were measured. A panoramic software programme was used to measure the ramus heights. Quantitative data was tested by Student's t test. Qualitative data was tested by $\chi^{2}$ test. The intraclass correlation coefficient was calculated for the magnitude error of the measurement.

Results: The mean of ramus asymmetry was found to be $2.90 \% \pm 2.58 \%$. Significant differences between the right and left ramus height ratios were observed ( $p<0.01)$. There was a high prevalence $(10.8 \%)$ of ramus asymmetry, which did not correlate with the age and gender of the patients.

Conclusions: This study revealed a high prevalence of ramus asymmetry in 9-21-year-old population. Within the limitations of this study, it can be concluded that ramus asymmetry should be carefully evaluated in all ages for the potential relation with temporomandibular dysfunctions and also for orthodontic anomalies. (Folia Morphol 2018; 77, 4: 724-729)

Key words: asymmetry, mandible, ramus, skull, anthropology, panoramic radiographs

\section{INTRODUCTION}

Facial asymmetry is characterised by differences in the two sides of the face in terms of size, form, and arrangement of facial landmarks [17]. Although perfect facial symmetry does not exist in nature [29], there is no consensus regarding the boundary between normal and pathological asymmetry [30]. Mandibular asymmetry, which is the primary cause of facial asymmetry, can be caused by variations in the height of the ramus or condyle [18].
A variety of aetiological factors have been reported in relation to facial asymmetry such as gender, age, facial growth pattern, functional and pathological alteration, dental occlusal changes and muscular activity [1, 21, 24, 27, 29]. Congenital changes such as hypoplasia of the ramus and condyle can play a role in the development of mandibular asymmetry [10]. Pathological factors such as infections, tumours, osteoarthritis of the temporomandibular joint, rheumatoid arthritis, and myogenic problems such 
as myospasm, chronic muscle shortening, muscle splinting, or occlusal interferences can also lead to mandibular asymmetry $[10,29]$. Trauma during the growth period can result in condylar asymmetries by disturbing the down-and-forward growth potential of the mandible [10].

Along with morphological asymmetry, functional and mechanical stresses may influence mandibular asymmetry [6]. Mongini et al. [14] showed that the chewing forces during mastication indicate the magnitude of joint loading over time, which is related to condylar size. el-Mofty [4] found that a reduction in the height of the mandibular ramus was associated with a decrease in function. The adaptive response of the mandible to deviations during function may cause remodelling of the condyle and glenoid fossa, which may lead to mandibular asymmetries [19].

Mandibular asymmetry is of great interest for both orthodontists and prosthodontic specialists, not only for aesthetic considerations, but also because of its involvement in the stomatognathic system which may cause functional problems such as temporomandibular disorders $[4,5,8,10,14,25,28]$. Several studies $[4,11-13,15,22]$ have described the relationship between condyle-ramus asymmetries and temporomandibular disorders. Internal derangement of the temporomandibular joint and osteoarthritis have been suggested as factors contributing to altered skeletal morphology $[15,16]$. A reduced ramus height and increased gonial angle have been reported to be associated with disc displacement and osteoarthritis [3]. Vertical asymmetries of the mandible have been used to diagnose temporomandibular disorders [11].

Ramus height measurements can provide a close approximation of ramus asymmetries. Various imaging modalities can be used to determine ramus, condyle and facial asymmetries, including clinical examinations, frontal and side view photography, lateral and posteroanterior cephalography, oblique radiography of the mandible at $45^{\circ}$, panoramic radiography, and three-dimensional computed tomography (3D CT) $[6,29,30]$.

A review of the literature revealed no studies comparing ramus asymmetry at growth spurts during puberty. Therefore, the purpose of this study was to determine the prevalence of ramus asymmetries in a 9- to 21-year-old population and to determine the possible influence of growth spurts on ramus asymmetries.

\section{MATERIALS AND METHODS}

The panoramic radiographs of 776 patients (441 females, 335 males, with an age range 9-21 years) who underwent clinical examination for dental indications were included in the study. Patients with a history of trauma, congenital craniofacial disease, systemic diseases, severe skeletal malocclusions, or previous orthodontic treatment were excluded. This retrospective study was conducted according to the principles of the Declaration of Helsinki. Written informed consent was obtained for all patients prior to imaging with panoramic radiograph.

The selected individuals were divided into two groups. The first group consisted of female subjects between 9 and 11 years of age and male subjects between 9 and 13 years of age, whereas the second group consisted of female subjects between 12 and 21 years of age and male subjects between 14 and 21 years of age.

Panoramic radiographs with no artefacts and particularly clear and visible condylar heads were chosen. One operator obtained the radiographs using Planmeca Promax system (Planmeca OY, Helsinki, Finland). All radiographs were obtained in a standard manner according to the manufacturer's operating instructions with the teeth in maximal intercuspation and the head oriented in the Frankfurt horizontal plane.

Bilateral ramus heights were measured from each digital radiograph with the aid of a panoramic software programme (Planmeca Dimaxis Pro software version 3.2.3; Planmeca OY, Helsinki, Finland). One dentist performed all image measurements. Two points were selected on the panoramic radiograph, one of which corresponds to the most lateral point of the condyle $\left(\mathrm{O}_{1}\right)$ and the other corresponding to the most lateral point of the angulus $\left(\mathrm{O}_{2}\right)$. Then a tangent line (A) was drawn connecting these two points. The distance between these points $\left(\mathrm{O}_{1}\right.$ and $\left.\mathrm{O}_{2}\right)$ on the tangent was calculated in millimetres as the ramus height using the software programme and recorded (Fig. 1A, B). The right and left side measurements for each radiograph were converted into a ratio using the following formula described by Habets et al. [6], revealing the degree of asymmetry.

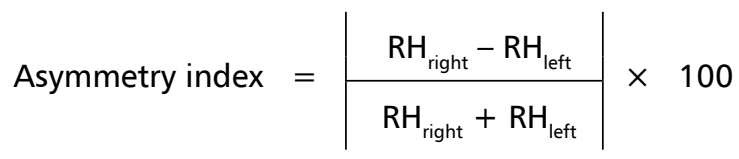

The asymmetry index formula gives results ranging from $0 \%$ to $100 \%$. Habets et al. [6] suggested that 


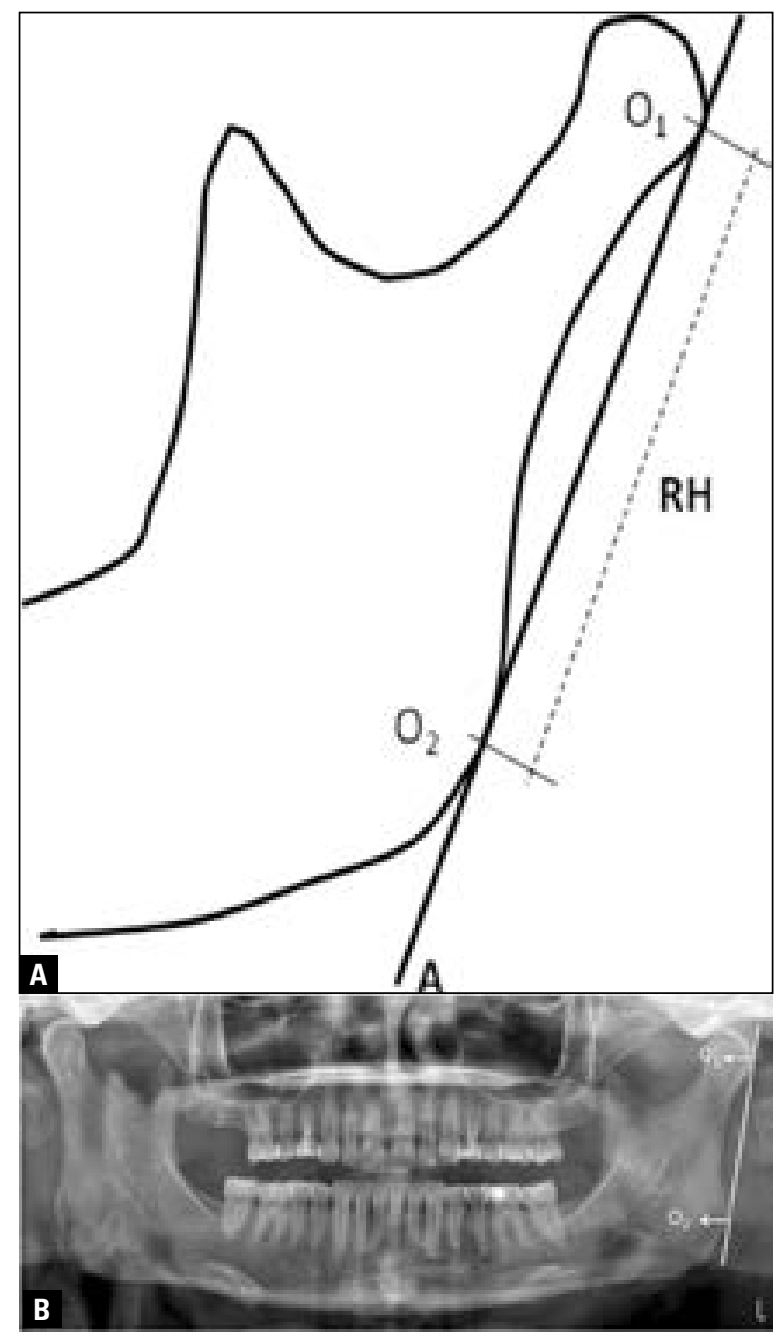

Figure 1. A. Schematic image of mandibular ramus; $A$ - ramus tangent; $O_{1}$ - the most lateral point of the condyle; $O_{2}-$ the most lateral point of angulus; $\mathrm{RH}$ - the distance between $\mathrm{O}_{1}$ and $\mathrm{O}_{2}$ (ramus height); $\mathbf{B}$. Measurement of ramus height (the distance between $\mathrm{O}_{1}$ and $\mathrm{O}_{2}$ ) on panoramic radiograph.

ramus height differences greater than $6 \%$ can be considered true vertical ramus asymmetries, as differences less than $6 \%$ might be a result of technical errors during measurement on panoramic radiographs.

\section{Statistical analysis}

Statistical analysis was performed using Number Cruncher Statistical System 2007 and Power Analysis and Sample Size 2008 (NCSS 2007 and PASS 2008 Statistical Software, NCSS LLC, Utah, USA). Quantitative data (mean asymmetry index evaluations according to gender and age) were assessed by Student's t-test. Qualitative data (numbers and percentages for the asymmetry index range and asymmetry index ratios according to gender and age) were assessed by a $\chi^{2}$ test. The intraclass correlation coefficient (ICC) was calculated to determine the magnitude of the measurement error. Results with $p<0.05$ were considered significant.

\section{Calculation of methodical error}

To calculate the magnitude of measurement error, 75 randomly selected panoramic radiographs were measured twice at a 1-month interval by the same investigator. The data were then subjected to a multiple regression analysis to determine an intraclass correlation estimate. The intraclass correlation analysis is shown in Table 1. The upper and lower limits of the confidence interval are provided in the table. The ICC values for all measurements were approximately 1.00. Thus, the methodical error can be considered negligible.

\section{RESULTS}

The analysis group comprised 776 patients, including 335 male patients and 441 female patients. The subjects' ages ranged from 9 to 21 years, with a mean age of $15.05 \pm 2.89$ years. The patients' asymmetry index results ranged from 0 to 24.51. The mean ramus asymmetry value was determined as $2.90 \pm 2.58$. Group 1 consisted of 168 (21.7\%) patients. Group 2 consisted of $608(78.3 \%)$ patients. There was a high prevalence $(10.8 \%)$ of ramus asymmetries in the studied population. However, statistically significant differences between the left and right ramus height ratios were observed for the entire study population on panoramic radiographs $(p<0.01$; Table 2$)$. No significant age- or gender-related differences were observed (Table 3). For male subjects with an asymmetry index less than 6 , asymmetry values ranged between 0 and 5.95 , with a mean of $2.26 \pm 1.62$. For male subjects with an asymmetry index of 6 or greater, asymmetry values ranged between 6.16 and 24.51, with an average of $8.55 \pm 3.43$. For female subjects with an asymmetry index less than 6 , asymmetry values ranged between 0 and 5.92, with a mean of $2.24 \pm 1.62$. For female subjects with an asymmetry index of 6 or greater, asymmetry values ranged between 6 and 19.35, with a mean of $8.08 \pm 2.37$.

Student's t-test was used to evaluate the mean asymmetry values $(\mathrm{mm})$ according to gender and age (Table 4). The mean asymmetry indices showed no gender- or age-related differences $(p>0.05)$.

\section{DISCUSSION}

The present study did not prove a relation between ramus asymmetry and age, gender. Females were more 
Table 1. Evaluation of method error for right and left ramus height measurements

\begin{tabular}{lcc}
\hline & $\begin{array}{c}\text { Intraclass correlation } \\
\text { coefficient }\end{array}$ & $\begin{array}{c}\text { 95\% confidence } \\
\text { interval }\end{array}$ \\
\hline Right ramus & 0.986 & $0.978-0.991$ \\
Left ramus & 0.994 & $0.981-0.992$ \\
\hline
\end{tabular}

Table 2. The number of subjects and percentages for the asymmetry index (Al) range using the $\chi^{2}$ test

\begin{tabular}{lccc}
\hline Al & Number & Percentage & P \\
\hline$<6$ & 692 & 89.2 & $0.001^{*}$ \\
$\geq 6$ & 84 & 10.8 & \\
\hline
\end{tabular}

${ }^{*}$ Significant $(p<0.01)$

Table 3. Distribution of asymmetry index ratios according to gender and age using the $\chi^{2}$ test

\begin{tabular}{|c|c|c|c|c|}
\hline & & \multicolumn{2}{|c|}{ Asymmetry index ratios } & \multirow[t]{2}{*}{$\mathbf{P}$} \\
\hline & & $<6$ & $\geq 6$ & \\
\hline \multirow{2}{*}{ Gender } & Male & $302(90.1 \%)$ & $33(9.9 \%)$ & \multirow{2}{*}{0.447} \\
\hline & Female & $390(88.4 \%)$ & $51(11.6 \%)$ & \\
\hline \multirow{2}{*}{ Age } & Group 1 & $151(89.9 \%)$ & $17(10.1 \%)$ & \multirow{2}{*}{0.739} \\
\hline & Group 2 & $541(89.0 \%)$ & $67(11.0 \%)$ & \\
\hline
\end{tabular}

$p>0.05$

Table 4. Distribution of mean asymmetry values according to gender and age

\begin{tabular}{llcc}
\hline & & Asymmetry values $[\mathrm{mm}]$ & P \\
\hline \multirow{2}{*}{ Gender } & Male & $2.88 \pm 2.65$ & 0.848 \\
& Female & $2.91 \pm 2.54$ & \\
\multirow{2}{*}{ Age } & Group 1 & $2.94 \pm 2.85$ & \multirow{2}{*}{0.818} \\
& Group 2 & $2.89 \pm 2.51$ & \\
\hline
\end{tabular}

Data are shown as mean \pm standard deviation. Student $t$ test used; $p>0.05$

likely to have asymmetry of ramus height compared to males although not significant. Similar results were found in other studies $[9,22,23]$ where dimensional mandibular asymmetries were reported to be independent of age and gender. However, some other studies $[10,12,13]$ showed correlations between condylar asymmetry and age. Sop et al. [24] showed that moderate or severe ramus asymmetry and asymmetry of the total ramus height in class III were more often found in males rather than in female subjects; thus males having moderate or significant overall asymmetry.

Although skeletal asymmetry exists to a certain extent in every human face, mandibular asymmetries should be considered important for both aesthetic reasons and for their potential associations with temporomandibular disorders. Thus, various studies have focused on the relationship between temporomandibular disorders and the orthodontic problems caused by condyle-ramus asymmetry, but no study has investigated the prevalence of ramus asymmetry alone or its associations with growth spurts. Miller et al. [12] investigated the relationship between condylar asymmetry in temporomandibular disorder patients aged between 13 and 42 years, and arthrogenous origin of pain. The authors concluded that condylar asymmetry decreased in increasing patient age. This was explained by the gradual loss of mesenchymal cells on the articular surface, which results in a reduction in asymmetry with the development of degenerative joint disease. Matilla et al. [10] also showed that asymmetry in male subjects with psoriatic arthritis seemed to decrease with increasing age. Research has shown that activation of the growth axis results in a linear growth spurt at around 12 years of age in girls and 14 years of age in boys [2]. In the present study, ramus asymmetry was seen in all ages. The different group age ranges could be the reason of different results of various studies.

In the dental literature, there are some studies $[3,8,10,19,20,28]$ investigating ramus-condyle asymmetries with sample sizes between 28 and 400 . However, this study included a higher number of cases (776 subjects) than those reported in the literature, and is expected to yield more reliable results.

In this study, panoramic radiographs were used to evaluate the prevalence of ramus asymmetry. Various imaging modalities can be used to determine ramus asymmetries, including clinical examinations, frontal and side view photography, lateral and posteroanterior cephalography, oblique radiography of the mandible at $45^{\circ}$, panoramic radiography, and 3D CT $[6,29,30]$. CT has been a method that is recently used for the evaluation of facial asymmetry and their relations to temporomandibular disorders. However, the radiation dose, which is higher than conventional radiography, brings some issues about its daily use and might be of ethical concern.

A noteworthy issue regarding the assessment of condyle-ramus asymmetries by panoramic radiographs is that, while the condyle structure is considered to be superimposed on the lateral edge of the glenoid fossa and the root of the zygomatic arch, the ramus can be easily visualised on a radiograph. This fact enables the 
use of panoramic radiographs to compare the vertical heights of the two rami [26]. Another important issue regarding panoramic radiographs is the magnification of structures on radiographic images in both vertical and horizontal directions $[26,28]$. Additionally, changes in head positioning can easily cause image distortions [20]. Horizontal measurements have been shown to be particularly unreliable because of non-linear variations in magnification at different object depths. On the other hand, many authors have considered panoramic radiographs adequate to perform vertical measurements when they are obtained using improved techniques and ideal head positioning with respect to the equipment (i.e., without a rotation or shift) [30]. In order to minimise distortions, all the subjects' heads were oriented in a standardised position under the same imaging conditions in our study. Additionally, the same operator using the same setup for all subjects obtained the radiographs. The magnification factor of panoramic radiographs is considered important when comparing exact linear measurements. Within the limitations of this study, panoramic radiographs were used to assess the asymmetry ratio between the two rami based on the fact that the percentages of right and left ramus height differences were compared using an asymmetry index rather than comparing exact linear measurements. Besides, considering using imaging techniques such as computed tomography for 776 patients would bring about ethical questions regarding the high radiation doses.

Different reference points for mandibular ramus height measurements have been used in various studies. Habets et al. [6] described ramus height as the distance between the most lateral points on the mandibular ramus tangent. However, other researchers $[7,25]$ measured ramus height between the incisura and gonion, which is the perpendicular distance between the deepest point of the mandibular notch and the lower border of the mandible. As the method developed by Habets et al. [6] has been preferred in most studies $[8,11,14,28]$, this measurement method was also used in our study.

The ramus or condyle height can be measured by a tracing on acetate paper, as described by Habets et al. [6]. Another method of measurement is via digitised panoramic radiographs. The perceived advantages of digitised radiographs include accurate determinations of bony structure contours resulting from the ability to enlarge the image and change the contrast as needed [7]. In addition, when a programme is used to measure ramus heights, it eliminates the risk of measurement error resulting from hand measurements. In this study, digitised panoramic radiographs were used to measure ramus heights.

\section{CONCLUSIONS}

Within the limitations of this study, a high prevalence $(10.8 \%)$ of ramus asymmetry in a population 9-21 years of age was revealed. The number of patients with ramus asymmetry was observed more frequently in females and ages between 14 and 21 . However, the difference was not statistically significant. Further controls in the post pubertal period can be conducted for the subjects with ramus asymmetries to find out whether there is compensation. Patients in whom ramus asymmetries are detected at early ages can be encouraged to come to control visits in order to determine whether the asymmetry will have any adverse clinical impact or result in a temporomandibular disorder.

\section{REFERENCES}

1. Arieta-Miranda JM, Silva-Valencia M, Flores-Mir C, et al. Spatial analysis of condyle position according to sagittal skeletal relationship, assessed by cone beam computed tomography. Prog Orthod. 2013; 14: 36, doi: 10.1186/21961042-14-36, indexed in Pubmed: 24325842.

2. Blakemore SJ, Burnett S, Dahl RE. The role of puberty in the developing adolescent brain. Hum Brain Mapp. 2010; 31(6): 926-933, doi: 10.1002/hbm.21052, indexed in Pubmed: 20496383.

3. Cho BH, Ahn YW, Jung YH. Comparison of mandibular morphology between patients with temporomandibular joint osteoarthritis and asymptomatic normal subjects. Quintessence Int. 2009; 40(8): e49-e54, indexed in Pubmed: 19639082.

4. el-Mofty S. Surgical correction of mandibular underdevelopment. Egypt Dent J. 1978; 24(3): 219-234, indexed in Pubmed: 389609.

5. Gidarakou IK, Tallents RH, Kyrkanides S, et al. Comparison of skeletal and dental morphology in asymptomatic volunteers and symptomatic patients with bilateral disk displacement with reduction. Angle Orthod. 2002; 72(6): 541-546, doi: 10.1043/0003-3219(2002)072<0541:COSADM > 2.0.CO;2, indexed in Pubmed: 12518945.

6. Habets LL, Bezuur JN, Naeiji M, et al. The orthopantomogram, an aid in diagnosis of temporomandibular joint problems. II. The vertical symmetry. J Oral Rehabil. 1988; 15(5): 465-471, indexed in Pubmed: 3244055.

7. Kjellberg H, Ekestubbe A, Kiliaridis S, et al. Condylar height on panoramic radiographs. A methodologic study with a clinical application. Acta Odontol Scand. 1994; 52(1): 43-50, indexed in Pubmed: 8184679.

8. Kurt G, Uysal T, Sisman Y, et al. Mandibular asymmetry in class II subdivision malocclusion. Angle Orthod. 2008; 78(1): 32-37, doi: 10.2319/021507-73.1, indexed in Pubmed: 18193958. 
9. Leversha J, McKeough G, Myrteza A, et al. Age and gender correlation of gonial angle, ramus height and bigonial width in dentate subjects in a dental school in Far North Queensland. J Clin Exp Dent. 2016; 8(1): e49-e54, doi: 10.4317/jced.52683, indexed in Pubmed: 26855706.

10. Mattila M, Könönen M, Mattila K. Vertical asymmetry of the mandibular ramus and condylar heights measured with a new method from dental panoramic radiographs in patients with psoriatic arthritis. J Oral Rehabil. 1995; 22(10): 741-745, indexed in Pubmed: 8606331.

11. Miller VJ. The effect of age on condylar asymmetry in patients with craniomandibular disorders of arthrogenous origin. J Prosthet Dent. 1992; 67(6): 845-846, indexed in Pubmed: 1403875.

12. Miller VJ, Myers SL, Yoeli Z, et al. Condylar asymmetry and its relation to age in a group of patients with a craniomandibular disorder of myogenous origin. J Oral Rehabil. 1994; 21(6): 707-711, doi: 10.1111/j.1365-2842.1994. tb01186.x, indexed in Pubmed: 7830206.

13. Miller VJ. Condylar asymmetry and handedness in patients with temporomandibular disorders. J Oral Rehabil. 1997; 24(7): 549-551, indexed in Pubmed: 9250844.

14. Mongini F, Preti G, Calderale PM, et al. Experimental strain analysis on the mandibular condyle under various conditions. Med Biol Eng Comput. 1981; 19(5): 521-523, indexed in Pubmed: 7334857.

15. Nebbe B, Major PW, Prasad NG, et al. TMJ internal derangement and adolescent craniofacial morphology: a pilot study. Angle Orthod. 1997; 67(6): 407-414, doi: 10.1043/0003-3219(1997)067<0407:TIDAAC>2.3. CO;2, indexed in Pubmed: 9428958.

16. Nebbe B, Major PW, Prasad NG. Female adolescent facial pattern associated with TMJ disk displacement and reduction in disk length: part I. Am J Orthod Dentofacial Orthop. 1999; 116(2): 168-176, indexed in Pubmed: 10434090.

17. Peck S, Peck L, Kataja M. Skeletal asymmetry in esthetically pleasing faces. Angle Orthod. 1991; 61(1): 43-48, doi: 10.1043/0003-3219(1991)061<0043:SAIEPF>2.0. CO;2, indexed in Pubmed: 2012321.

18. Pirttiniemi PM. Associations of mandibular and facial asymmetries: a review. Am J Orthod Dentofacial Orthop. 1994; 106(2): 191-200, doi: 10.1016/S08895406(94)70038-9, indexed in Pubmed: 8059759.

19. Ramirez-Yañez GO, Stewart A, Franken E, et al. Prevalence of mandibular asymmetries in growing patients. Eur J Orthod. 2011; 33(3): 236-242, doi: 10.1093/ejo/cjq057, indexed in Pubmed: 20724557.
20. Raustia AM, Salonen MA. Gonial angles and condylar and ramus height of the mandible in complete denture wearers--a panoramic radiograph study. J Oral Rehabil. 1997; 24(7): 512-516, doi: 10.1111/j.1365-2842.1997. tb00366.x, indexed in Pubmed: 9250838.

21. Saccucci M, D'Attilio M, Rodolfino $D$, et al. Condylar volume and condylar area in class I, class II and class III young adult subjects. Head Face Med. 2012; 8: 34, doi: 10.1186/1746-160X-8-34, indexed in Pubmed: 23241136.

22. Sağlam AM. The condylar asymmetry measurements in different skeletal patterns. J Oral Rehabil. 2003; 30(7): 738-742, indexed in Pubmed: 12791161.

23. Saglam AA, Sanli G. Condylar asymmetry measurements in patients with temporomandibular disorders. J Contemp Dent Pract. 2004; 5(3): 59-65, indexed in Pubmed: 15318257.

24. Sop I, Mady Maricic B, Pavlic A, et al. Biological predictors of mandibular asymmetries in children with mixed dentition. Cranio. 2016; 34(5): 303-308, doi: 10.1080/0 8869634.2015.1106809, indexed in Pubmed: 27077261.

25. Staudt CB, Kiliaridis S. Association between mandibular asymmetry and occlusal asymmetry in young adult males with class III malocclusion. Acta Odontol Scand. 2010; 68(3): 131-140, doi: 10.3109/00016350903460182, indexed in Pubmed: 20085500.

26. Türp JC, Vach W, Harbich K, et al. Determining mandibular condyle and ramus height with the help of an orthopantomogram: a valid method? J Oral Rehabil. 1996; 23(6): 395-400, indexed in Pubmed: 8809694.

27. Ueki K, Nakagawa K, Marukawa K, et al. The relationship between temporomandibular joint disc morphology and stress angulation in skeletal class III patients. Eur J Orthod. 2005; 27(5): 501-506, doi: 10.1093/ejo/cji029, indexed in Pubmed: 16024561.

28. Uysal T, Sisman Y, Kurt G, et al. Condylar and ramal vertical asymmetry in unilateral and bilateral posterior crossbite patients and a normal occlusion sample. Am J Orthod Dentofacial Orthop. 2009; 136(1): 37-43, doi: 10.1016/j. ajodo.2007.06.019, indexed in Pubmed: 19577146.

29. Van Elslande DC, Russett SJ, Major PW, et al. Mandibular asymmetry diagnosis with panoramic imaging. Am J Orthod Dentofacial Orthop. 2008; 134(2): 183-192, doi: 10.1016/j. ajodo.2007.07.021, indexed in Pubmed: 18675198.

30. Yañez-Vico RM, Iglesias-Linares A, Torres-Lagares D, et al. Diagnostic of craniofacial asymmetry. Literature review. Med Oral Patol Oral Cir Bucal. 2010; 15(3): e494-e498, indexed in Pubmed: 20038905. 